\author{
Journal of Measurement, Electronic, Communication, and Systems (2016) SP0216-01 \\ www.jmecs.org/content/vol2
}

\title{
Spectrum Sensing in Cognitive Radio Using Combined Sequential Energy Detector and Cyclostationary Feature Detector
}

\author{
S. Poundel ${ }^{1}$, H. Wijanto ${ }^{1}$ and F. Y. Suratman ${ }^{1}$ \\ ${ }^{1}$ School of Electrical Engineering, Telkom University, Bandung, 40287, Indonesia
}

\begin{abstract}
In the following research, we derive a detector which is based on sequential probability ratio test (SPRT) and it uses Energy Detector (ED) which is followed by Cyclostationary Feature Detector (CFD). ED is a blind sensing technique and it is easy to implement while conceptually simple. However, it is highly affected by interference and noise uncertainties. Therefore, CFD is applied for fine sensing as research has shown that Cyclostationary Feature Detector is more suitable than the energy detection when noise uncertainties are unknown. Our method is novel in trying to derive a sequential Energy Detector and combine it with Cyclostationary Feature Detector for low SNR region where average sample number (ASN) as a random variable may take very high value to achieve a desired performance level for sequential Energy Detector. For this sequential Energy Detector is terminated after it reaches certain cutoff sample number, making it truncated sequential Energy Detector.
\end{abstract}

Keywords: Cognitive Radio, Primary User, Secondary User, Sequential Probability Ratio Test, Average Sample Number

\section{Introduction}

Increasing number of applications and wireless devices have increased demand for radio spectrum and they are leading to the problem of spectrum scarcity. Recent research has shown that most of the available spectrum is either underutilized or completely idle which can be seen in a particular case in figure 1. Federal Communications Commission (FCC) published a report prepared by Spectrum Policy Task Force in November 2002, which reports that [1]: In many bands, spectrum access is a more significant problem than physical scarcity of spectrum, in large part due to legacy command-and-control regulation that limits the ability of potential spectrum users to obtain such access. The case of spectrum underutilization can be improved if a secondary user can access the unused licensed spectrum bands during a certain temporal frame of time in which it is unoccupied. Therefore,
Opportunistic Spectrum Access (OSA) has been proposed as a solution for present problem of spectrum scarcity and the new spectrum policy is called Dynamic Spectrum Allocation (DSA) policy, whereby the secondary or non-licensed users can sense the frequency bands and if it is found that the bands are not being used at that particular time, they start communicating using those bands. This characteristically, is a feature of Cognitive Radio [2] that is touted as a revolution in the future of wireless communication which attempts at Dynamic Spectrum Allocation.

The name mostly refers to spectrum aware communication systems. It is defined as Software Defined Radio which is aware of its environment, it learns from and has the ability to change its parameters according to these changes in its environment and the network requirements [3]. 
Poudel et al. / Journal of Measurement, Electronics and Communication Systems (2016) SP0216-01

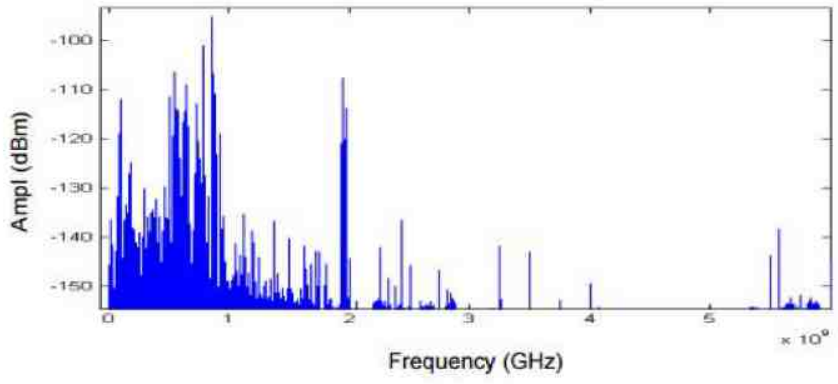

Fig. 1. A Snapshot of the spectrum utilization up to $6 \mathrm{GHz}$ in an urban area [16]

The term cognitive radio (CR) refers to a radio that is aware of its surrounding environment and it can intelligently access the unused spectrum bands thereby increasing the communication capacity and efficiency. Once such empty spaces are found, it is possible for secondary users who do not hold license or right to the frequency band. Hence, for the efficient use of spectrum the first step is to sense whether the spectrum is being used by primary users or not. One of the most important cognitive features of the cognitive radio standard is spectrum sensing which senses the white spaces or channels void of primary users in the available frequency spectrum. Spectrum sensing has been viewed as a signal detection problem whereby spectrum sensors detect the presence of primary user signals. Most of the spectrum sensing algorithms are based on sample size and sensing time which are predetermined and fixed. In contrast, Wald proposed that a detector based on sequential detection requires less average sensing time in comparison to a fixed sample size detector. In paper [4], the authors propose an intelligent spectrum sensing scheme which improves the utilization efficiency of the radio spectrum by increasing detection reliability and decreasing sensing time. The scheme chooses either the combined energy and cyclostationary detector or the matched filter detection depending upon the suitability. In this paper [5], the authors maintain that Cyclostationary Feature Detector is a good method for PU detection in cognitive radio systems but long detection time leads to inefficient spectrum utilization. They propose to apply sequential analysis to the Cyclostationary Feature Detector to improve on sensing delay. In paper [6], the authors propose an Energy Detector based on sequential probability ratio test to reduce the average required sample number and sensing time for spectrum sensing in lowsignalto-noise ratio regime. Data samples are grouped into blocks and the sequential probability ratio test uses energy of the block as test statistic. In [7], the authors propose a two-stage spectrum sensing where coarse sensing is performed by Energy Detector and fine sensing is performed by Cyclostationary Feature Detector. Threshold parameters are derived in such a way as to maximize the probability of detection under constraints on probability of false alarm. We propose a detector which is based on Sequential Probability Ratio Test (SPRT) and uses combined energy as well as Cyclostationary Feature Detector. Sequential analysis will decrease the sensing time which carries a huge significance in spectrum sensing. Energy Detector as a non-coherent detection method does not require any prior knowledge of a PUs waveform and so is easy to implement, however it is highly affected by interference and noise uncertainties [8]. Therefore, Cyclostationary Feature Detector is applied for a reliable sensing accuracy in low SNR as the researches have shown that Cyclostationary Feature Detector is more suitable than the energy detection when the noise uncertainties are unknown [9]. In general, test statistic is calculated using SPRT for Energy Detector, terminated after the sample number for the test reaches certain predetermined value and further analyzed using Cyclostationary Feature Detector to perform the task of sensing presence of primary user signals. Combined spectrum sensing technique of ED and CFD has been used to obtain better results with less mean detection time [4]. Our method is novel in trying to derive a sequential Energy Detector and combine it with Cyclostationary Feature Detector for low SNR region where average sample number (ASN) as a random variable may take very high value to achieve a desired performance level for sequential Energy Detector. For this sequential Energy Detector is terminated after it reaches certain cut-off sample number, making it truncated sequential Energy Detector.

Decision whether a channel is free or not is based on the result of a binary hypothesis testing experiment [9]. In signal detection, the task is to decide whether observation were generated under null or alternative hypothesis [10]. This binary hypothesis testing problem in spectrum sensing is formulated as a decision between two hypotheses $\mathrm{H} 0$ and $\mathrm{H} 1$, where $\mathrm{H} 0$ is the null hypothesis and $\mathrm{H} 1$ is the alternative hypothesis. If the decision is $\mathrm{H} 0$ hypothesis then it means the absence of primary user signal. However, H1 decision means the presence of 
primary user signal in the spectrum. Hypotheses can be formulated as:

$$
\begin{aligned}
& H_{0}: y[n]=w[n] \\
& H_{1}: y[n]=s[n]+w[n]
\end{aligned}
$$

where $\mathrm{h}$ is the channel gain, $\mathrm{x}[\mathrm{n}]$ is the primary signal and $\mathrm{w}[\mathrm{n}]$ is the additive noise. The detector collects the received samples, builds a test statistic and compares it against predefined thresholds.

\section{A. Detection Problem}

Probability of False Alarm (PF A): The decision is made on presence of primary user signal when the spectrum band is actually unoccupied.

$$
P_{F A}=P\left(>\lambda \mid H_{0}\right)=P\left(u=1 \mid H_{0}\right)
$$

Probability of Miss Detection (PMD): the decision is made on absence or primary user signal when the frequency band is actually occupied.

$$
P_{M D}=P\left(<\lambda \mid H_{1}\right)=P\left(u=0 \mid H_{1}\right)
$$

Probability of Detection (PD): The decision is made on presence of primary user signal when the frequency band is actually occupied.

$$
P_{D}=1-P_{M D}=P\left(u=1 \mid H_{1}\right)
$$

\section{Detector}

\subsection{Energy Detector}

This is the simplest form of spectrum sensing and this detector decides whether the primary signal is resent based on comparison of measured energy against a given threshold and whether it exceeds that threshold. The predetermined threshold is highly susceptible to the noise floor, in-band interferences, and channel notches caused by frequency selective fading. The decision metric, $\mathrm{Y}$, is built from a sequence of $\mathrm{N}$ received signal samples:

$$
Y=\frac{1}{N} \sum_{n=0}^{N-1}|y|_{n}^{2}
$$

We assume noise to be an i.i.d random Gaussian process with zero mean and variance $2 / \mathrm{n}$.
The primary users signal is considered to be an i.i.d random process with zero mean and variance $2 / \mathrm{s}$.

$$
\begin{aligned}
& H_{0}: y[n]=w[n] \\
& H_{1}: y[n]=s[n]+w[n]
\end{aligned}
$$

where $\mathrm{n}=0,1 . . \mathrm{N}-1$ For Neyman Pearson detector, it decides the alternative hypothesis $H_{l}$ if the likelihood ratio exceeds the value of predetermined threshold.

$$
L(y)=\frac{p\left(y ; H_{1}\right)}{p\left(y ; H_{0}\right)}>\lambda
$$

Likelihood ratio can be further given as

$$
L(y)=\frac{\left[2 \pi\left(\sigma_{n}^{2}+\sigma_{s}^{2}\right]^{\exp }\left[\frac{1}{2\left(\sigma_{n}^{2}+\sigma_{s}^{2}\right)} \sum_{n=0}^{N-1} y[n]^{2}\right]\right.}{\frac{1}{\left[2 \pi\left(\sigma_{n}^{2}\right)\right]^{\frac{N}{2}}} \exp \left[-\frac{1}{2\left(\sigma_{n}^{2}\right)} \sum_{n=0}^{N-1} y[n]^{2}\right]}
$$

If we take the log-likelihood ratio we get

$$
l(y)=\frac{N}{2} \ln \left(\frac{\sigma_{n}^{2}}{\sigma_{n}^{2}+\sigma_{s}^{2}}\right)+\frac{1}{2}\left(\frac{\sigma_{s}^{2}}{\sigma_{n}^{2}\left(\sigma_{n}^{2}+\sigma_{s}^{2}\right.} \sum_{n=0}^{N-1} y[n]^{2}\right)
$$

Hence the decision is $H_{l}$ if

$$
T(y)=\frac{1}{N} \sum_{n=0} N-1 y[n]^{2}>\lambda
$$

The test statistic $\mathrm{T}(\mathrm{y})$ for large number of samples can be modeled by a Gaussian distribution as follows [11], $T(y) \sim N\left(\sigma_{n}^{2},\left(2 \sigma_{n}^{4}\right) / N\right)$ under $H_{0}$ and $T(y) \sim N\left(\left(\sigma_{n}^{2}+\sigma_{s}^{2}\right), 2\left(\sigma_{n}^{2}+\sigma_{s}^{2}\right)^{2} / N\right)$ under $H_{l}$.

Probability of false alarm $P_{F A}$ is given by [12]

$$
P_{F A}=Q\left(\left(\frac{\lambda}{\sigma_{n}^{2}}-1\right) \sqrt{\frac{N}{2}}\right)
$$

where $Q$ is the complementary distribution function of the standard Gaussian, $\lambda$ is the threshold and $\mathrm{N}$ is the number of samples. For predetermined values of $P_{F A}$ and $P_{D}$ the required number of samples to achieve such performance level is given by [12].

$$
N=\frac{2}{\gamma^{2}}\left[Q^{-1}\left(P_{F A}\right)-Q^{-1}\left(P_{D}\right)(\gamma+1)\right]^{2}
$$




\subsection{Cyckistationary Feature Detector}

If a signal is not completely known but some of its features are known then we can use the Cyclostationary Feature Detector approach to detect the presence of primary user signal. Generally, modulated signals exhibit some form of periodicity which means that signal is not completely stationary but cyclostationary. However, the additive noise is considered to be stationary and hence this difference in between signal of interest and noise can be used to detect the presence of a signal of interest. This technique works very well even during the low SNR and is not hampered by the noise uncertainty unlike in the case of Energy Detector. Sometimes it is easier to analyze signals in the frequency domain than in the time domain. Analyzing signals in the frequency domain lets us to detect the presence or absence of signals during the process of spectrum sensing. As we have already mentioned about the cyclic autocorrelation function now we can mention that spectral correlation density is the Fourier transform of CAF. The SCD is given by

$$
S_{x}^{\alpha}(f)=\int_{-\infty}^{\infty} R_{x}^{\alpha}(\tau) e^{(-i 2 \pi f \tau)} d t
$$

for a continuous signal and $x(t)$ and by

$$
S_{x}^{\alpha}(f)=\int_{k=-\infty}^{\infty} R_{x}^{\alpha}[k] e^{(-i 2 \pi f k)}
$$

for a discrete signal $x[n]$.

\subsection{Sequential Probability Ratio Test}

Individual detectors have fixed sample sizes. However, new hypotheses tests have been proposed which do not use fixed sample size which on average need a smaller sample sizes to achieve the same PD and PF A. These include the sequential test proposed by A. Wald [15]. The sensing time is a random variable, which on average is less than that for the fixed sample detector for the same performance. Performance evaluation of Sequential detection can be done in terms of Average sample Number (ASN), which is the number of samples on average required to make a decision at a given level of accuracy during a binary hypothesis testing [13].

The ASN function is the expected value of random stopping time $\mathrm{N} s$ of the SPRT when the underlying distribution is $f(y[n] ; \theta), \theta \in \Theta$. By using the same argument that the increment under $\mathrm{z}^{-}$under $f\left(y_{N} ; \theta\right)$ is very small compared to A - B, the ASN function can be approximated by [14]

$$
E_{H}\left[N_{s}\right]=\frac{\left(\left(1-e^{t B}\right) A+\left(e^{t A}-1\right) B\right)}{\left(\left(e^{t A}-e^{t B}\right) E_{H}[z]\right)}
$$

where, $E_{H}$ denotes the expected value under $f(x[n] ; \theta)$ and $z$ denotes the increment per sample. The ASN under $H_{0}$ and $H_{l}$ can be found from above equation by substituting $\mathrm{t}=1$ and $\mathrm{t}=-1$, respectively [15]. The increment $z$ using the distribution shown above and in [11] for the $n_{t h}$ sample $Y_{n}$ can be given as

$$
z=\ln \frac{1}{(1+\gamma)}-\frac{L}{4 \sigma_{n}^{4}}\left[\frac{1}{(1+\gamma)^{2}}\left(Y_{n}-(1+\gamma) \sigma_{n}^{2}\right)^{2}-\left(Y_{n}-\sigma_{n}^{2}\right)^{2}\right]
$$

where $\mathrm{L}$ is the number of received samples that is used to calculate the test statistic for the sequential Energy Detector. Main purpose of using sequential Energy Detector over using Energy Detector is because of its advantage in lowering average number of samples required for the detector to achieve a given performance level. However, ASN of SED is a random variable and especially in the low SNR region it requires higher number of samples. Moreover, as SED is based in conventional Energy Detector it is prone to noise uncertainties and in-band interference in the low SNR region. Hence, we propose CFD as the second stage of sensing for the low SNR values. Analytically, we can determine the sample number that is acceptable to us at a particular SNR value and we can switch to the CFD when the decision is still not made between the null and alternative hypothesis.

\subsection{Truncated Sequential Energy Detector}

The reason for applying sequential test to the binary hypothesis testing problem is to save the detection time and lower the number of samples that is required to come to a decision. Average sample numbers for sequential testing can be very large especially at the lower SNR region and stringent constraint. To save ourselves from high detection time which is directly proportional to the sample numbers required to come to a decision, we choose another criterion for termination of the test. We take a reasonable predetermined cut-off sample number 
Poudel et al. / Journal of Measurement, Electronics and Communication Systems (2016) SP0216-01

and if the detector cannot make the decision until even when the cut-off sample number is reached we terminate the sequential testing and move forward to the use of Cyclostationary Feature Detector. This method of sequential testing is called truncated sequential testing and hence the sequential Energy Detector can also be called as truncated sequential Energy Detector in such condition. Probability of detection for the combined detector is given by [21] as

$$
P_{D}=P_{D}^{c}+\left(1-P_{D}^{c}\right) P_{D}^{f}
$$

And probability of false alarm for the combined detector is given by [21] as

$$
P_{F A}=P_{F A}^{c}+\left(1-P_{F A}^{c}\right) P_{F A}^{f}
$$

where, $P_{D}$ is the overall probability of detection, $P_{F A}$ is the overall probability of false alarm, $P_{F A}{ }^{c}$ and $P_{D}{ }^{c}$ are the probabilities of false alarm and detection for coarse sensing respectively and $P_{F A}{ }^{f}$ and $P_{D}{ }^{f}$ are the probabilities of false alarm and detection for coarse sensing respectively.

\section{Simulation and Results}

Digitally modulated QPSK signal is considered to be the signal of interest. In figures 2 and 3 , we compare the required number of sample numbers for both ED and SED. In both cases we can see that the numbers of samples required to attain a given performance level is lower in the case of Sequential Testing based Energy Detector than in individual detector which in our case is Energy Detector. We consider two cases: firstly, with predetermined values of $P_{F A}$ and $P_{M D}$ as 0.01 and secondly, with the values as 0.05 . For, the case of $P_{F A}$ and $P_{M D}$ both equal to 0.01 we can see that the average number of samples is more than halved.

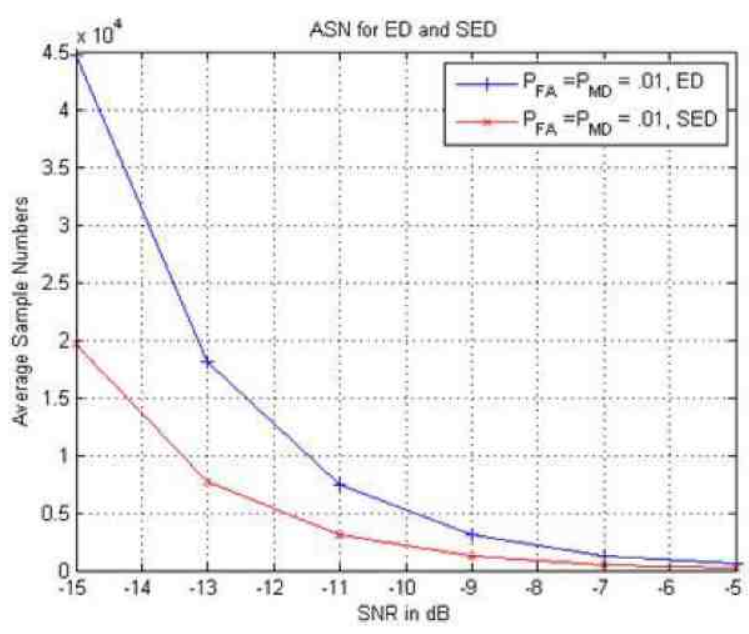

Fig. 2. ASN for different values of SNR for SED and ED, $P_{F A}=P_{M D}=0.01$

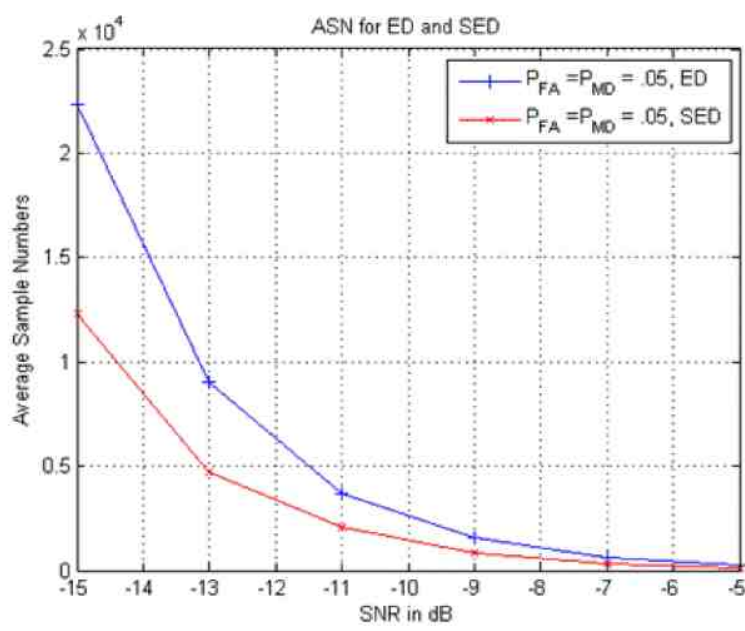

Fig. 3. ASN for different values of SNR for SED and ED, $P_{F A}=P_{M D}=0.05$

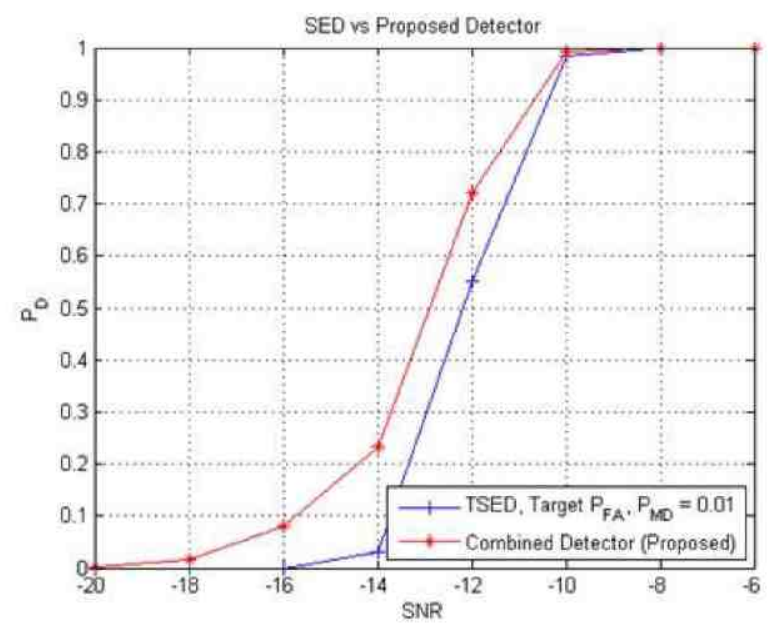

Fig. 4. Performance comparison of truncated SED and Combined Detector

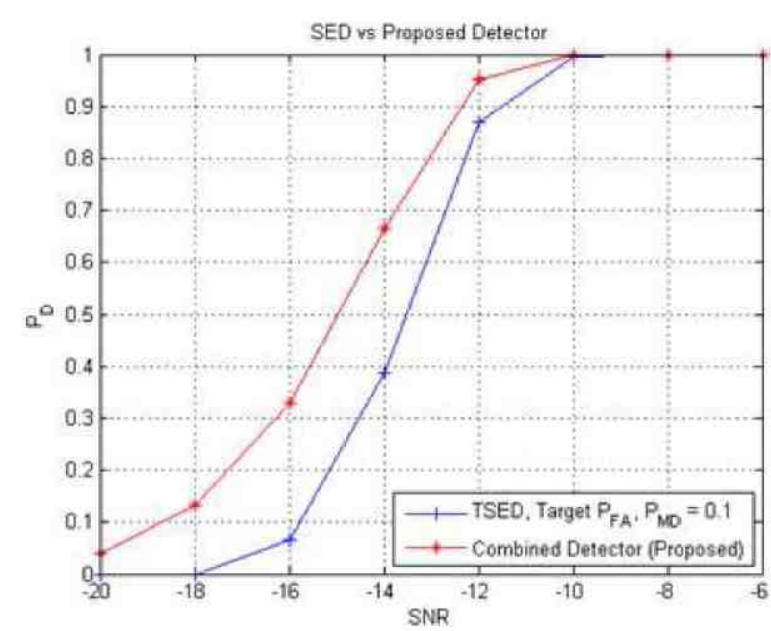

Fig. 5. Performance comparison of truncated SED and Combined Detector

In figure 4 and 5, we show that using combined detectors as proposed we can improve the performance over sequential Energy Detector. For the simulation we use the cut-off sample number for SED as 5000 and if the detector cannot detect the presence of signal in that condition we move forward 
to Cyclostationary Feature Detector. We have taken two different target constraints. From the experiment we can see that there has been increase in the performance of the combined detector than when only using one detector.

\section{Conclusions}

In this thesis, our work has been to focus on reducing the sensing time in a $\mathrm{CR}$ system using sequential detection. Our sequential detection is based on Energy Detector and we further analyze the signal using Cyclostationary Feature Detector. By using sequential Energy Detector we have compared it to traditional Energy Detector to show that the average sample number decreases in the formers case. Also we have shown that using combined detectors as proposed we can improve the performance over sequential Energy Detector. For the simulation we the cut-off sample number for SED as 5000 and if the detector cannot detect the presence of signal in that condition, we move forward to Cyclostationary Feature Detector. There are many areas that might be useful for further research. We may consider performance evaluation of the proposed detectors in different kinds of fading channels like log-normal shadowing channel and Rayleigh fading channel. One might consider using sequential probability ratio test for cyclostationary spectrum sensing as well. And we hope that better to have truncated sequential Energy Detector before analyzing the received signal with Cyclostationary Feature Detector. Furthermore, collaborative spectrum sensing can be used after the individual combined detectors to solve the problem like hidden terminal and it will also increase the sensing accuracy.

\section{Reference}

[1]Federal Communications Commission, "Spectrum Policy Task Force", Rep. ET Docket no. 02-135, Nov. 2002.

[2]Mitola, Joseph, and Gerald Q. Maguire Jr. "Cognitive radio: making software radios more personal." Personal Communications, IEEE 6.4 (1999): 13-18.

[3] Haykin, Simon. "Cognitive radio: brainempowered wireless communications. Selected Areas in Communications, IEEE Journal on 23.2 (2005): 201-220.
[4] W. Ejaz, N. U. Hasan, S. Lee, and H. S.Kim, "I3S: Intelligent spectrum sensing scheme for cognitive radio networks," EURASIP J. Wirel. Commun. Netw., vol. 2013, no. 1, p. 26, 2013

[5] Choi, Kae Won, Wha Sook Jeon, and Dong GeunJeong. "Sequential Detection of Cyclostationary Signal for Cognitive Radio Systems."

[6] Wireless Communications, IEEE Transactions on 8.9 (2009): 4480-4485. X. Zhang and Z. Qiu. ”A Sequential Energy Detection Based Spectrum Sensing Scheme in Cognitive Radio", Research Journal of Applied Sciences, Engineering and Technology 5(3): 1002-1007, 2013

[7] Maleki, Sina, Ashish Pandharipande, and Geert Leus. "Two-stage spectrum sensing for cognitive radios." Acoustics Speech and Signal Processing (ICASSP), 2010 IEEE International Conference on. IEEE, 2010.

[8] Luo, Ling, et al. "A two-stage sensing technique for dynamic spectrum access." Wireless Communications, IEEE Transactions on 8.6 (2009): 3028-3037.

[9] T. Yucek and H. Arslan, "A survey of spectrum sensing algorithms for cognitive radio applications," Commun. Surv. Tutorials, IEEE, vol. 11, no. 1, pp. 116130, 2009.

[10] E. Axell, G. Leus, E. Larsson, and H. V. Poor, "Spectrum sensing for cognitive radio," Proc. IEEE, no. MAY 2012, pp. 101116, 2009.

[11] D. Cabric, A. Tkachenko and R.W. Brodersen, "Spectrum sensing measurements of pilot, energy, and collaborative detection", IEEE MILCOM, Oct. 2007.

[12] Liang, Ying-Chang, Yonghong Zeng, Edward CY Peh, and Anh Tuan Hoang. "Sensingthroughput tradeoff for cognitive radio networks." Wireless Communications, IEEE Transactions on 7, no. 4 (2008): 1326-1337

[13] A. Wald, "A Sequential Analysis", John Wiley \& Sons, New York, 1947

[14] Abraham Wald, "Sequential Analysis", Columbia University, 1945 
Poudel et al. / Journal of Measurement, Electronics and Communication Systems (2016) SP0216-01

[16] Suratman, Fiky Y., "Spectrum Sensing in Cognitive Radio: Bootstrap and Sequential Detection Approaches",

FachgebietSignalverarbeitung, Darmstadt, [Ph.D.

Thesis], (2014

[17] R. W. Broderson, A. Wolisz, D. Cabric, S. M. Mishra and D. Willkomm, "White paper: corvus: a cognitive radio approach for usage of virtual unlicensed spectrum," Tech. Rep, 2004. 